\title{
XXVI. Memoir upon the mordants employed in the art of dyeing
}

\section{Thenard \& Roard}

To cite this article: MM. Thenard \& Roard (1812) XXVI. Memoir upon the mordants employed in the art of dyeing , Philosophical Magazine Series 1, 39:167, 175-186, DOI: 10.1080/14786441208638108

To link to this article: http://dx.doi.org/10.1080/14786441208638108

曲 Published online: 27 Jul 2009.

Submit your article to this journal

Џ Article views: 2

Q View related articles 
XXVI. Memoir upon the Mordants employed in the Art of Dyeing. By MM. ThEnard and Roard. Read at the Physical and Mathematical Class of the French Institute.

T HE name of Mordant is given, in the art of dyeing, to those substances which serve to produce a more intimate combination of colouring matters with the different stuffs, and to augment the brightness and beauty of them. This property belongs to a great number of saline and metallic substances : but those which possess it in the highest degree, and which, for this reason, are exclusively made use of by dyers, are alum, acetate of alumina, tartar, and the solutions of tin.

An examination and analysis of the effects produced by these mordants upon vegetable and animal substances, will form the subject of the memoir we have honour to submit to the Class. We shall divide it into four chapters, wherein we shall successively treat of the artion of alum, of acetate of alumina, of alum and tartar, and of the solutions of tin, upon silk, wool, cotton, and thread, according to the methods must generally made use of in the art of dyeing.

\section{CHAP. I.-Of Alum.}

The manner of applying the alum varies according to the nature of the stuffs, and according to the colours we wish to obtain. Silks are permitted to macerate for several days in a solution of alum, sufficiently diluted for the salt not to crystallize. Wool is boiled for two hours in water, containing a fourth part of its weight of alum. Cotton and thread are soaked for at least twenty-four hours in warm concentrated solutions or alum, to which frequently some potass is added. It has hitherto been thought that in this operation the alum is decomposed, and that the alumina combines with the stuff, causing it thereby the more easily to take the colour when plunged into the dyeing bath; but the experiments we have made induce us to adtopt a different opinion.

ArT. 1.-Analysis of the aluming of Silk.

Ninety-five grammes of silk, well cleaned and perfectly purified, were infused in a glass vessel during six days, at the common temperature of the atmosphere, with four quarts of distilled water, containing 100 grammes of pure alum, which had been previously dissolved in it. After standing 
standing this time, the silk was taken out of the liquid, drained completely over the bath, and washed several times with distilled water, to separate that part of the mordant which had not combined with it. The alum bath and the washings were then evaported with the greatest care, and they afforded very transparent crystals of alum. These first products indicated pretty clearly the nature of the combination which had been formed with the silk during the steeping, and that the alum had not been decomposed. The alumed silk was then boiled in a mattras with six quarts of distilled water, the boiling liquor was poured off from it, and in this manner it was treated twelve times. The 72 quarts proceeding from these operations being evaporated, we obtained well formed crystals of alum, the quantity of which, added to that obtained from the bath, amounted to within two decigrammes of the 95 grammes originally employed, forming a loss of $x^{\frac{1}{2}} \mathrm{c}$ part only. If after each of the twelve washings we attempt to dye the silk, the colour is less deep, in proportion to the number of washings, so that after the twelfth the silk is not at all coloured. If the silk, after having thus been washed, is again impregnated with alum, it re-acquires the same property of retaining the colour which it had before the washing commenced. Hence results a very natural explanation of the reason why alumed silks take a deeper colour when the dyeing is conmenced at a low temperature, than when they are plunged into boiling baths; it is because, in the one case, the action of the boiling water upon the mordant is so speedy, that there is not time for the colouring matter to fix upon it, and render the combination insoluble, whilst, in the other case, no such effect takes place.

\section{ArT. 2.-Analysis of the Impregnation of Wool with Alum.}

After having thus ascertained the phænomena which take place in the aluming silk, it was necessary to continue the trials upon wool, and to employ for these experiments only perfectly pure materials, completely deprived of the carbonate of lime, which is generally contained in considerable quantity. To separate the whole of this, we boiled the wool several successive times in a mattras with weak muriatic acid, but in order to take up the last portions of this acid; we were obliged to make use of such large quantities of distilled water, that we were on the point of abandoning such tedious experiments, requiring so much time and patience, as well as the greatest care. The separation of all the muriatic acid from the first two hectogrammes of 
wool which we purified, required 200 quarts of distilled water, at 100 degrees of heat $(212 \mathrm{~F}$.) divided in to 20 successive operations, each occupying from seven to eignt hours. When calcined and properly tried, it afforded neither lime nor muriatic acid.

One hundred grammes of this wool were alumed with the same care which had been taken with the silk. It was afterwards washed twenty times, employing six quarts of distilled water, heated to an hundred degrees, for each washing. Immediately after the aluming, this wool took a very deep colcur, whilst, after the last washing, it would not take any more colnur in the dyeing bath, than some of the same wool which had never been alumed. These experiments convinced us that the substance which had been fixed in the wool by aluming, and had caused it to receive so deep a colour in the first dycing, had now been carried off by the wattr. The alum bath, when evaporated, afforded us, in the state of crystals, two-thirds of the quantity of alum we had originally employed; very nearly the whole of the remaining third part was obtained from the residue of the bath, in an uncrystallized state, and from the washings of the wool. This experiment was repeated several times, and always with the same result; but as this did not appear to us so decisive as the experiment upon silk, on account of the difficulty of separating the animal malter from the last portions of the alum bath, we alumed some wool in the cold, as we had done with the silk, being persuaded that in this case the bath would not sensibly dissolve this substance.

We alumed in the cold, some clean wool with all the precaution observed with the silk, and we obtained from the bath and the washings the alum exployed in the operation, with a loss only of $\frac{1}{40}$. part; we were therefore assured, that in the aluining of all animal substances, the alum cumbines entirely with them, without undergoing any decomposition, and that it forms with them combinations more or less soluble, which have a great affinity for the colouring matters.

\section{Ant. 3.- Analysis of the Impregnation of Cotton and Thread uith Alum.}

Having freed some cotton, by the methods already mentioned, from all foreign matters, we macerated it for two days in a lukewarm solution of a given quantity of alum. After this operation the stuff took the dye remarkably well ; but being treated with boiling distilled water, it lost

Vol.39. No. 167. March $181 \%$. M the 
the property of taking the colour in the dye-bath. The alum-bath and washings, when evaporated, afforded us all the alum we had employed. We separated this alum from the vegetable matter which it had dissolved in its different crystallizations. To do this did not require such a number of washings as were employed for the wool or the silk, because the combination of alum with vegetable substances is so weak, that soaking the alumed cotton in boiling water for a few minutes is sufficient to carry off the greatest part of the mordant. Cotton, therefore, ought to be dyed at a low temperature, since it is only after the colouring matter has rendered the combination insoluble, that it can support a great heat without being attacked. Thread treated in the same manner as cotton, afforded us the same results.

\section{ArT. 4.-Analysis of the Impregnation of common Wool.}

The analyses we have already related, most decidedly dernonstrate that in the aluming of all animal and vegetable substances, the alum combines with them without undergoing any decomposition; but we thought it was necessary to repeat the same experiments upon these substances in the state in which they are commonly met with in commerce, as we had done in their purified state. Wool, when impregnated with alum alone, aiways renders the bath turbid, which, upon cooling, throws down an abundant white precipitate, as has been observed by scveral chemists. Several analyses of this sediment, after being well washed, have constantly afforded us some sulphate of lime, saturated sulphate of alumina, and sometimes a little alumina. The bath contained a remarkable quantity of alum, of acidulated sulphate of potass, combined with a small proportion of animal matter. Upon the wool we found alum, and a very minute proportion of the precipitate. These experiments upon the sediment, formed in the alum bath, do not differ from those made by $M$. Bcrthollet; but this learned chemist not having cxamined the mother-waters, nor the alumed wool, has not given, as he himself says, a clear and precise explanation of the effects produced by alum and tartar in the operation of dyeing. These precipitates, obtained by treating common wool with alum, never take place with purified wool; and as these only differ from each other by the former containing some carbonate of lime, it was natural in this case to attribute to this substance the decomposition of a part of the alum.

We satisfied ourselves of this, by mixing in glass vessels solutions 
solutions of alum in boiling water with different proporrions of pure carbonate of lime. We always found the alum was decomposed by the carbonate of lime, and that, if a sufficient quantity was added, there remained no part of the aluminons salt in solution. The mother-waters contained very acid sulphate of potass, and the sediment was formed of sulphate of lime and acidulate sulphate of alumina and potass; whence it follows, that the property possessed by common wool of forming a precipitate in the alum bath, and rendering the fluid very acid, is in reality owing to the carbonate of lime it contains. The same result was obtained by aluming common wool five or six successive times in the same bath. But in order to arrive at a general solution of this question, it was necessary to ascertain the nature of the precipitates formed in the soJution of alum, by different alkalue and earthy substances. We took, therefore, alums with base of potass, and with base of ammonia, which we treated with ammonia and carbonate of potass, so as to leave in the solution but a slight excess of alum. The mother-waters evaporated, contained very acid sulphates of ammonia, of potass and ammonia, and of potass, according to the nature of the alum and of the precipitate employed. The sediment, which was acid sulphate of alumina and potass, or ammonia, treated with sulphuric acid, afforded alum and acidulated sulphate of alumina; boiled afterwards a great number of times with distilled water, it was converted into alum, sulphate of potass, and pure alumina. There was always a greater quantity of acidulated sulphate of potass than of alum, even in the last washings.

Solutions of alum, treated at a boiling heat with pure alumina, were converted into a very acid sulphate of potass, and into acidulated sulphate of alumina and potass. These results do not at all correspond with those obtained by M. Vauquelin in his experiments upon the alums of commerce, for we have never been able to obtain the saturated sulphate of alumina and potass, spoken of by that celebrated chemist.

Thus all the alkaline and earthy substances mixed in suitable proportions with solutions of alum, converted that salt into acidulated sulphate of potass, or ammonia, and into insoluble acid sulpbate of alumina and potass or ammonia, for which reason we have named it the acidulated sulphate instead of the saturated sulphate, the name it bas borne until now. It is evident, that if too great a quantity MI 
of carbonate of lime be employed, and the same holds good with the carbonates of barytes and strontian, we shall only obtain alumina and the sulphates of potass, lime, barytes, or strontian. There remains no doubt, therefore, of the nature of the changes produced in the alum baths by the common wools, and of the prejudicial effects of alkalies in the baths intended for cottons; for the addition of these substances diminishes the quantity of alum, and even increases the acidity of the bath.

\section{CHAP. II.}

On the Impregnation of Vegetalle and Animal Matters with Acetate of Alumina.

Wool, silk, cotton, and thread, in the different states in which these substances are employed for dyeing, were treated with acetate of alumina, which combined entirely with them. But as in exposing them to the air, or to a temperature a little elevated, the mordant always loses a small quantity of acid, it follows that the combination formed upon the stuff is an acetate with excess of base; thus, by treating it with boiling water, it is converted into acidulated acetate of alumina, which is dissolved, and into alumina which cannot be carried off by the water.

CHAP. III.

ART. 1.-Of the Action of acidulated Tartrite of Potass on Wool.

Purified wool $u$ as treated as in the former experiments with very pure cream of tartar, free from tartrite of lime, and formed directly by the tartaric acid and potass. This wool was washed a great number of times, until the last washing did not contain any of the principles which had been combined with it. The bath afforded by evaporation $\frac{3}{4}$ of the crean of tartar employed, or rather neutral tartrite of potass. The washings were very acid, and we obtained from them a small quantity of cream of tartar, and a very acid composition formed of tartarous acid and wool. These facts may be thought sufficiently to explain the phænomena which take place in impregnating wool with alum and tartar, since we already know from the experiments of M. Berthollet, that these two salts are not decomposed; and as we have shown that the wool combincs completely with the alum, and that it acts upon the cream of tartar by separating the tartarous acid with which it unites in the most intimate manner. But in order to have these facts rigorously 
rigorously demonstrated, we repeated this experiment, although a very tedious one, in the method already pointed out in the preceding chapters.

\section{Art. 2.-Of the Action of Alum and Tartar upon Wool.}

Before treating the wool with alum and cream of tartar, we made some trials of the reciprocal action of these two salts. We ascertained that water, at the temperature of $12^{\circ}$ or $14^{\circ},\left(55 \mathrm{~F}\right.$.) holds in solution only $\mathrm{T}^{\prime} \frac{1}{30}$ part of its weight of cream of tartar, that boiling water dissolves $\frac{1}{30}$ of its weight, and that a mixture of equal parts of alum and cream of tartar, dissulves in $\frac{3}{5}$ the quantity of water required to dissolve the salts separately at the same temperature. These results do not differ from those already obtained by M. Berthollet, who has shown that alum has the property of increasing the solubility of cream of tartar.

If wool is alumed in the ordinary proportions, which are $\frac{1}{4}$ of the weight of the stulf of alum, and $\frac{1}{T}$ of cream of tartar, all the substances being perfectly pure, we obtain from the bath when evaporated, alum, cream of tartar, and a residue difficully crystallizable, composed of tartrite of potass and an animal matter; the washings of the wool will give alum, a small quantity, scarcely appretiable, of cream of tartar, and a very acid combination, formed of a large quantity of tantarous acid, alum, and animal matter.

These experiments remove all uncertainty concerning many practical facts, which at present are only noticed by the dyer in a vague way, and point out to him the precise merhod of applying the mordants according to the nature of the colour he wishes to obtain. Indeed, since by making use of alum and tartar, the wool is impregnated with alum and a large quantity of tartarous acid, these two salts should never be employed together, except when the colour is susceptible of being heightened and rendered brighter by acids, as is the case with cochineal, madder, and kermes. On the contrary, alum should never be employed for wools intended to be dyed with woad or Brasil wood, the colour of which is easily altered or destroyed by acids. Among all the vegetable and animal substances, we have made choice of wool only for trial with alum and alum and tartar, because it is only with this substance these mordants are made use of in dyeing.

Art. 3.- On the Action of Acids, and of some Salts em. ployed as Mordants upon $W_{\text {Wool. }}$

Although all researches hitherto marle have been inefM 3 fectual 
fectual to find a substitute for alum, we have, nevertheless, made trial of a great number of substances with wool, less, however, for the purpose of discovering the best mordants, than for determining the action of several substances, very soluble, and at the same time endowed with great powers. We boiled wool for two hours in water, in which were put small quantities of suphuric, nitric, muriatic, and tartaric acids. In each instance, the wool, especially when combined with sulphuric acid, struck with cochineal and madder deeper colours than when impregnated with alum and tartar. No doubt, therefore, can be entertained of their superiority in similar cases : but of all the mordants we tricd, there is not one which gives such bright colours as what are obtained by means of the acid tartrite of alumina (notwithstanding the opmion of M. Hausmann to the contrary). This salt w muld, in a great number of cases, be preferable to tartar and ahum, if ats price was not so much higher than theirs. Whilst we were occupied in inquiring with the greatest care into erery thing relative to the nature and mode of combination of mordants with various stuffs, we did not forget to examine the several methods which have been adopted in all the worksbops for a lung time past, in order to ascertain if the proportions of alum and tartar, the nost generally employed, were those the most suitable for the purpose, if the time employed for the alum bath was sutficicnt to impregnate the wool sufficiently, and if the exposure to the cool air afterwards, for several days, which is so generally thought necessary, is attended with the expected advantages.

Equal parts of the mordants, that is, half the weight of the suff; prodicer! no better effect than one-fourth; but between this quantity and one-twentieth part, the colours of cocinineal, kcrmes, and madder, were weaker in proportion to the diminution of the quantity of the salts; whilst, on the contrary, the effects were reversed with woad and Brazil wood, so that in these last substances, the colour was deeper the more the salts were dimiuished. No difference could be observed in the colour whether the wools had been in the alum bath for two, four, or six hours; it is, therefore, useless to continue stuffs in the bath longer than two hours. Our experiments did not discover that there was any difference in the colour, whether the dyeing took place inmediately after the aluming, or was protracted for sume time, except only that wool impregnated with alum alone, produced a deeper colour with woad, after baving been exposed some time to a cool air, which we 
attributed to the separation of the acidulated sulphate of potass, this being carried of with the uncombined mordant in drying.

\section{CHAP. IV. Art, 1.-Of the Scarlet Colour.}

Scarlet is that hright and shining colour which is produced in wool by treating it with tartar, cochineal, and a bighly oxidized solution of tin. Before the discovery of this method, for which we are entirely indebted tu Drebbel, those colours were called scarlet which are produced in woollen stuffs by kermes or cochineal, when alum and tartar are employed as mordants. These processes for obtaining this colour have long been known in the dyeing houses, yet no theoretic investigations have been made into the phrnomena whicls take place when a solution of tin is used with cream of tartar and cochineal. Dr. Bancroft attempted to explain what passes in the formation of this colour; but as his opinion does not appear to be founded on any experiments, we considered the question as not at all determined by his labours. We propose, therefore, in this fourth chapter, to determiue the chemical nature of the combination formed upon wool by cochineal, tartar, and a solution of tin, and to make known the result of our inquiries upon the coluur ot scarlet.

An'r. 2.- Examination of the Precipitate formed by the Solution of Tin, and the acidulated Tartrite of Potass.

All the substances employed by us in our experiments were perfectly pure, and we constantly made use of glass vessels and distilled water. Eighty grammes of acidulated tartrite of potass dissolyed in three kilogrammes, and five hectogrammes * of distlled water, were macerated for two hours, at 100 degrees $(212 \mathrm{~F}$.) of heat, with oue hundred and twenty-five grains of a solution of tin. The precipitate which we obtained was washed several times, and distilled in a small curred retort, the beak of which being plunged into lime water, there was disengaged a sensible quantity of carbonic acid. Proper re-agents indicated in other portions of it the presence of a great deal of tin and muriatic acid. Thus the cream of tartar and solution of tin are decomposed, and produce a precinitate, consisting of tartarous acid, and a great quantity of muriatic acid and tin. The mother-water contains tartrite of potass, acidulated tartrite, very acid muriate of tin, and a considerable

* About seven pints.

$\mathrm{M} 4$ 
portion of precipitate, held in solution by excess of muriatie acici.

Very pure white wool, treated with the ordinary proportions of solution of tan and cream of tartar employed in dyeing scarlet, was washed a great number of times in boiling water, which carried of all the substances combined with it. These washings, collected and evaporated, afforded us the same principles we had betore obtained from the precipitate formed by the solution of tin and cream of tartar; we also examined, in the same way, the action of cochineal, and found no difference in the results. From these facts we are to a certainty convinced, that the fine scarlet colour is produced by the wool being combined with colouring matter, tartarous acud, muriatic acid, and peroxide of tin. But we are mistaken, if we think the bath has no. infuence on the colour; for wool combined with the mordants we sball presently mention, and dyed with cochineal, never take the scarlet hue unless some acid be added, which causes the colour to pass from yellowish to red, and at length to a bright colour. This last experinent, and some others we sball relate towards the close of this memoir, proved to us that the wool is not coloured yellow by the combination it forms with the nitric acid in excess in the solution of tin, for this wiol comes out perfectly white from all the bollings it undergoes with the tin, when no colouring matter is employed.

\section{A Rт, 3.-Of Tartyites of Tin, and some other Metallic Solutions.}

The proofs we have alcady given of the formation of scarlet, appear to us so decisive, that we should not have thought of increasing the number, had not the importance of the question indnced us to extend further our labours on this sutject.

We ried upon wool, in the usual proportions for dyeing scarlet, ail the sulphates and muriates of antimony, bisinuth, zinc, and arsenic. Some of these solutions afforded very agrecable colour, but very different from that we were secking to obtain. We were more fortunate in our attempts with the tartrite of tin obtained from tartrate of potass and soda, and a highly oxidized muriate of tin. "This salt dissolved in muriatic acid, and used in the operation of dyeing, affordcd us a scarlet colour as beautiful and bright as those obtaincel by cream of tartar and a solution of tin. The tartrite of tin, also, dissolved in an excess of its own acid, produced very good eflects; however, as this 
method would be more costly than the ordinary processes, it is best to employ the solution of this salt in muriatic acid. But before recommending this mordant to be used in the dye houses, we intend to make trial of it in the large way, so as to determine precisely the experse of it, and what advantages will be obtained by its employment.

\section{AnT. 4.-Experiments upon the Colour of Scarlet and Oxides of Tin.}

Scarlet, as we bave already seen, is obtained by treating wool with determined proportions of cochineal, acidulated tartrite of potass, and a highly oxidized solution of t $\mathrm{n}$. The operation of dyeing is divided into two parts : the first taking up an hour and a half, the latcer half an hour; this division is necessary to produce a good colour, which would be weaker and more yellow if all the substances were mixed in the first operation, and applied to the wool for two hours. This circumstance is owing to the very acid state of the bath, which holds in solution a great part t) the mordant, and of the colouring matter. We obtain the contrary effect when the mordants only are employed in the first operation, and the cochineal reserved for the second.

Pieces of very beautiful scarlet cloth, macerated in distilled water, at a boiling heat, gave out to the water a portion of their colour, and when the operation was finished, appeared only of a light flesh colour. The washings collected and evaporated were very acid, and contained, besides the colouring substance and animal matter, tartarous acit, muriatic acid, and oxide of tim. Scarlet, thercfore, as we have already shown, is a combination in some measure soluble, which in parting with a small quantity of acid changes its shade, and may, by repeated washings at elevated temperatures, and with a large bulk of fluid, be rendered completely colourless.

It results from the experiments related in this memoir:

1st. That in aluming all regetable and animal substances, it is not the alumina which combines with them, but the entire alum; and that when these matters are not purified, the lime which they contain, occasions a decomposition of a part of this mordant.

2. That all the alkaline and earthy bases, mixed with a solution of alum, decompose it, and convert it into acid sulphate of potass, and into an insoluble salt, less acid than alum, which may, by repeated washings, be converted into pure alumina, sulphate of potass, and alum.

3. That the acetate of alumina combines also in its en- 
tire state with silk, wool, cotton, and thread; that this compound retains its acid but fcebly, and loses a portion of it by simple exposure to the air ; and that it is then changed into acid acetate of alimina, which is carried off by water, and into alumina which remains upon the stuffs.

4. That alum and tartar are not decomposed, but that the solubility of the latter is increased by the mixture; and that in impregnating wools either with tartar, or alum and tartar, the tartar alone is decomposed, that the tartarous acid and alum combine with the stuff, and tartrite of potass remains in the bath.

5. That the most powerful acids have the property, when combined with wool, of fixing the colouring matters, a property possessed in a high degree by the acid tartrite of alumina.

6. That alum and tartar cannot be employed indifferently for all colours, and that their proportions must depend upon the nature of the colouring matter; that the time of aluming should not be more than two hours, and that the exposure of the stuffs in a moist place, after the mordants are applied, is of no utility in augmenting the intensity of their colour.

7. That highly oxidized tartrite of in, dissolved in $\mathrm{mu}-$ riatic acid, may supply the place of cream of tartar and the solution of tin in dyeing scarlet.

8. Lastly, that these experiments furnish some useful hints for combining mordants with the stuffs to be dyed, and for improving several of the processes of dyeing.

To complete these researches relative to the action of mordants, it would without doubt be necessary to determine, in the most accurate manner, the changes produced in these combinations by the colouring matters, when applied to the different stuffs; but these experiments, which we have already commenced, will form the subject of a second Memoir, to be hereafter presented to the Class.

XXVII. An Account of the Smelting of Lead. By Mra JOHN SADLER.

[Continued from vol. xxxviii. p. 376.]

" Crpola Smelting*.

I He process of smelting here described, appears to be defective in some points, which I will take the liberty to mention, and at the same time suggest the means of im-

* See an account of the present state of this art, in Mr. Farey's Derbyshire Report, vol. i, p. 386.-EDITor.

improvement : 